\title{
274.
}

\section{DEUXIEME NOTE SUR LA TRANSFORMATION DE TSCHIRNHAUSEN.}

[From the Journal für die reine und angewandte Mathematik (Crelle), tom. LvIII. (1861), pp. 263-269.]

À LA fin de ma première note sur ce sujet j'ai appliqué la transformation de Tschirnhausen à l'équation du troisième degré mise sous la forme

$$
a x^{3}+3 b x^{2}+3 c x+d=\left(a, b, c, d \gamma(x, 1)^{3}=0 .\right.
$$

En y substituant $\frac{1}{3} b, \frac{1}{3} c$ au lieu de $b, c$, cette équation se change en

$$
a x^{3}+b x^{2}+c x+d=\left(a, b, c, d \gamma(x, 1)^{3}=0,\right.
$$

et en même temps le résultat obtenu dans ma première note s'énonce de la manière suivante :

En calculant pour l'équation

la transformée en

$$
\left(a, b, c, d^{8}(x, 1)^{3}=0,\right.
$$

$$
y=\left(a x+\frac{1}{3} b\right) T_{0}+\left(a x^{2}+b x+\frac{2}{3} c\right) T_{1},
$$

on obtient

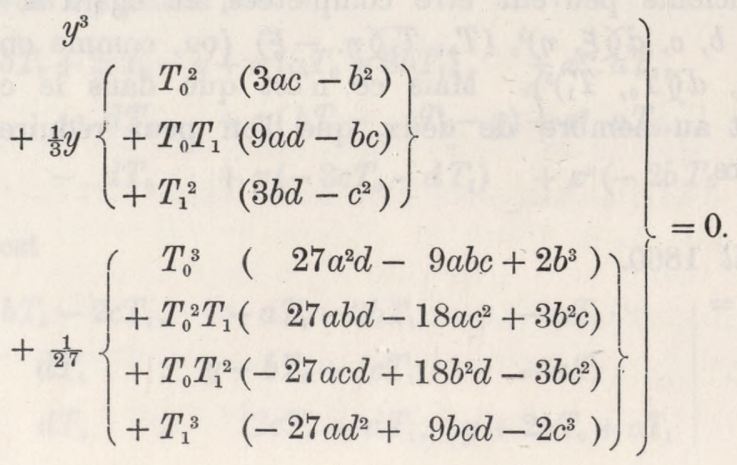


Je vais me servir de cette formule, pour en déduire l'équation qui d'une manière analogue est la transformée de l'équation du quatrième ordre

J'écris d'abord

$$
\left(a, b, c, d, e^{\gamma}(x, 1)^{4}=0 .\right.
$$

$$
\begin{gathered}
\left(a, b, c, d, e^{\gamma}(x, 1)^{4}=0,\right. \\
y=\left(a x+\frac{1}{4} b\right) T_{0}+\left(a x^{2}+b x+\frac{1}{2} c\right) T_{1}+\left(a x^{3}+b x^{2}+c x+\frac{3}{4} d\right) T_{2},
\end{gathered}
$$

et je remarque qu'en faisant $e=0$, le système proposé se partage en deux, dont le premier est:

et le second:

$$
\begin{aligned}
& x=0, \\
& y=\frac{1}{4}\left(b T_{0}+2 c T_{1}+3 d T_{2}\right),
\end{aligned}
$$

$$
\begin{gathered}
\left(a, b, c, d^{\gamma}(x, 1)^{3}=0,\right. \\
y=\left(a x+\frac{1}{4} b\right) T_{0}+\left(a x^{2}+b x+\frac{1}{2} c\right) T_{1}-\frac{1}{4} d T_{2} ;
\end{gathered}
$$

ou, ce qui est la même chose,

$$
\begin{gathered}
\left(a, b, c, d \gamma(x, 1)^{3}=0\right. \\
y+\frac{1}{12}\left(b T_{0}+2 c T_{1}+3 d T_{2}\right)=\left(a x+\frac{1}{3} b\right) T_{0}+\left(a x^{2}+b x+\frac{2}{3} c\right) T_{1} .
\end{gathered}
$$

Une circonstance analogue a lieu dans l'équation en $y$, résultat de l'élimination $d u$ système proposé. Pour $e=0$ son premier membre se résout de même en deux facteurs qui égalés à zéro sont les résultats de l'élimination du premier et du second système ci-dessus écrits. Le premier de ces deux facteurs est donc

$$
y-\frac{1}{4}\left(b T_{0}+2 c T_{1}+3 d T_{2}\right)
$$

et le second (en vertu de la formule donnée antérieurement)

$$
\begin{aligned}
& \left\{y+\frac{1}{12}\left(b T_{0}+2 c T_{1}+3 d T_{2}\right)\right\}^{3} \\
+\frac{1}{3}\left[\left(3 a c-b^{2}\right) T_{0}{ }^{2}+\text { etc. }\right] & \left\{y+\frac{1}{12}\left(b T_{0}+2 c T_{1}+3 d T_{2}\right)\right\} \\
+\frac{1}{27}\left[\left(27 a^{2} d-9 a b c+2 b^{3}\right)\right. & \left.T_{0}{ }^{3}+\text { etc. }\right]
\end{aligned}
$$

donc en multipliant les deux facteurs, et en égalant à zéro leur produit, on a la transformée en $y$ de la forme $(a, b, c, d, 0\} x, 1)^{4}$. Or dans le cas général, où $e$ est différent de zéro, les coefficients de la transformée en $y$ sont des invariants des deux formes

$$
\left(a, b, c, d, e^{\gamma} \chi \xi, \eta\right)^{4}, \quad\left(T_{0}, T_{1}, T_{2} \gamma \eta,-\xi\right)^{2} .
$$

Cette propriété permet de déduire leurs valeurs générales des valeurs particulières qu'ils ont pour $e=0$. Je formerai de cette manière la transformée en $y$ pour la forme $\left(a, b, c, d, 0^{\gamma} \gamma x, 1\right)^{4}$, je passerai de là à la forme $\left(a, b, c, d, 0 \gamma(x, 1)^{4}\right.$ (ce qui se fait en écrivant $4 b, 6 c, 4 d$ au lieu de $b, c, d)$, et enfin je complèterai les valeurs des coefficients en y introduisant $e$ au moyen de la propriété que doivent posséder les coefficients d'être des invariants des deux formes

$$
\left(a, b, c, d, e^{\gamma} \xi, \eta\right)^{4}, \quad\left(T_{0}, T_{1}, T_{2} \gamma \eta,-\xi\right)^{2} .
$$

On obtient d'abord l'équation en $y$ sous la forme

$$
(1,0,(5, D),(E) y, 1)^{4}=0 \text {, }
$$

c. IV. 
où

$$
\begin{aligned}
& 8 \widetilde{c}=\quad T_{0}^{2}\left(8 a c-3 b^{2}\right) \\
& +T_{0} T_{1}(24 a d-4 b c) \\
& +T_{1}^{2}\left(8 b d-4 c^{2}\right) \\
& +T_{0} T_{2}(-2 b d) \\
& +T_{1} T_{2}(-4 c d) \\
& +T_{2}^{2}\left(-3 d^{2}\right) \text {, } \\
& 8 D=\quad T_{0}^{3} \quad\left(8 a^{2} d-4 a b c+1 b^{3}\right) \\
& +T_{0}{ }^{2} T_{1} \quad\left(\quad 4 a b d-8 a c^{2}+2 b^{2} c\right) \\
& +T_{0} T_{1}^{2} \quad\left(-16 a c d+4 b^{2} d\right) \\
& +T_{1}^{3}\left(-8 a d^{2}\right) \\
& +T_{0}^{2} T_{2} \quad\left(-4 a c d+1 b^{2} d\right) \\
& +T_{0} T_{1} T_{2}\left(-12 a d^{2}\right) \\
& +T_{1}^{2} T_{2} \quad\left(-4 b d^{2}\right) \\
& +T_{0} T_{2}{ }^{2} \quad\left(-1 b d^{2}\right) \\
& +T_{1} T_{2}^{2} \quad\left(-2 c d^{2}\right) \\
& +T_{2}^{3}\left(-1 d^{3}\right) \\
& 256 \&=\quad T_{0}^{4}\left(64 a^{2} b d+16 a b^{2} c-3 b^{4}\right) \\
& +T_{0}^{3} T_{1} \quad\left(-128 a^{2} c d-80 a b^{2} d+64 a b c^{2}-8 b^{3} c\right) \\
& +T_{0}{ }^{2} T_{1}{ }^{2} \quad\left(-128 a b c d+64 a c^{3}-48 b^{3} d+8 b^{2} c^{2}\right) \\
& +T_{0} T_{1}^{3} \quad\left(\quad 64 a b d^{2}+64 a c^{2} d-128 b^{2} c d+32 b c^{3}\right) \\
& +T_{1}^{4}\left(128 a c d^{2}-64 b c^{2} d+16 c^{4}\right) \\
& +T_{0}^{3} T_{2} \quad\left(-192 a^{2} d^{2}+32 a b c d-4 b^{3} d\right) \\
& +T_{0}^{2} T_{1} T_{2}\left(-288 a b d^{2}+64 a c^{2} d+8 b^{2} c d\right) \\
& +T_{0} T_{1}^{2} T_{2}\left(-160 b^{2} d^{2}+48 b c^{2} d\right) \\
& +T_{1}^{3} T_{2} \quad\left(+192 a d^{3}-128 b c d^{2}+32 c^{3} d\right) \\
& +T_{0}{ }^{2} T_{2}{ }^{2} \quad\left(-48 a c d^{2}+14 b^{2} d^{2}\right) \\
& +T_{0} T_{1} T_{2}^{2}\left(-144 a d^{3}+8 b c d^{2}\right) \\
& +T_{1}^{2} T_{2}^{2} \quad\left(-48 b d^{3}+8 c^{2} d^{2}\right) \\
& +T_{0} T_{2}^{3} \quad\left(-\quad 4 b d^{3}\right) \\
& +T_{1} T_{2}^{3} \quad\left(-1 c d^{3}\right) \\
& +T_{2}^{4}\left(-8 d^{4}\right) \text {. }
\end{aligned}
$$

Ce calcul achevé et substituant la forme $(a, b, c, d, 0 \gamma \xi, \eta)^{4}$ au lieu de $(a, b, c, d, 0 \gamma \xi, \eta)^{4}$ (ou $4 b, 6 c, 4 d$ au lieu de $b, c, d$ ) on obtient tous les termes de l'équation cherchée, hormis ceux qui contiennent $e$ : et ces derniers s'obtiennent au moyen de ce que les coefficients des différentes puissances de $y$ se réduisent à zéro par l'opérateur

$$
a \partial_{b}+2 b \partial_{c}+3 c \partial_{d}+4 d \partial_{e}-T_{2} \partial_{T_{1}}-2 T_{1} \partial_{T_{i}}
$$


Cela ne présente pas de difficulté, je supprime donc les calculs intermédiaires et je donne le résultat final que voici: les équations

$$
\begin{gathered}
\left(a, b, c, d, e \gamma(x, 1)^{4}=0,\right. \\
y=(a x+b) T_{0}+\left(a x^{2}+4 b x+3 c\right) T_{1}+\left(a x^{3}+4 b x^{2}+6 c x+3 d\right) T_{2},
\end{gathered}
$$

conduisent à la transformée :

$$
\left(1,0,\left(5, D,(E)(y, 1)^{4}=0\right.\right.
$$

\begin{tabular}{|c|c|c|c|c|c|c|}
\hline & $T_{0}^{2}$ & $T_{0} T_{1}$ & $T_{0} T_{2}$ & $T_{1}^{2}$ & $T_{1} T_{2}$ & $T_{2}^{2}$ \\
\hline$=$ & $\begin{array}{l}a c+3 \\
b^{2}-3\end{array}$ & $\begin{array}{l}a d+6 \\
b c-6\end{array}$ & $\begin{array}{l}a e+2 \\
b d-2\end{array}$ & $\begin{array}{l}a e+1 \\
b d+8 \\
c^{2}-9\end{array}$ & $\begin{array}{l}b e+6 \\
c d-6\end{array}$ & $\begin{array}{c}c e+3 \\
d^{2}-3\end{array}$ \\
\hline
\end{tabular}

\begin{tabular}{|c|c|c|c|c|c|c|c|c|c|c|}
\hline & $T_{0}^{3}$ & $T_{0}{ }^{2} T_{1}$ & $T_{0}^{2} T_{2}$ & $T_{0} T_{1}^{2}$ & $T_{0} T_{1} T_{2}$ & $T_{1}^{3}$ & $T_{0} T_{2}{ }^{2}$ & $T_{1}^{2} T_{2}$ & $T_{1} T_{2}^{2}$ & $T_{2}^{3}$ \\
\hline 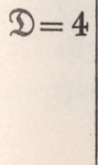 & $\begin{array}{l}a^{2} d+1 \\
a b c-3 \\
b^{3}+2\end{array}$ & $\begin{array}{l}a^{2} e+1 \\
a b d+2 \\
a c^{2}-9 \\
b^{2} c+6\end{array}$ & $\begin{array}{l}a b e+1 \\
a c d-3 \\
b^{2} d+2\end{array}$ & $\begin{array}{l}a b e+4 \\
a c d-12 \\
b^{2} d+8\end{array}$ & $\begin{array}{l}a d^{2}-6 \\
b^{2} e+6\end{array}$ & $\begin{array}{l}a d^{2}-4 \\
b^{2} e+4\end{array}$ & $\begin{array}{l}a d e-1 \\
b c e+3 \\
b d^{2}-2\end{array}$ & $\begin{array}{l}a d e-4 \\
b c e+12 \\
b d^{2}-8\end{array}$ & $\begin{array}{l}a e^{2}-1 \\
b d e-2 \\
c^{2} e+9 \\
c d^{2}-6\end{array}$ & $\begin{array}{l}b e^{2}-1 \\
c d e+3 \\
d^{3}-2\end{array}$ \\
\hline
\end{tabular}

où l'on $\mathrm{a}$

\begin{tabular}{|c|c|c|c|c|c|c|c|c|}
\hline & $T_{0}^{4}$ & $T_{0}{ }^{3} T_{1}$ & $T_{0}{ }^{3} T_{2}$ & $T_{0}^{2} T_{1}^{2}$ & $T_{0}{ }^{2} T_{1} T_{2}$ & $T_{0} T_{1}^{3}$ & $T_{0}{ }^{2} T_{2}{ }^{2}$ & $T_{0} T_{1}^{2} T_{2}$ \\
\hline \& & $\mid \begin{array}{l}a^{3} e+1 \\
a^{2} b d-4 \\
a b^{2} c+6 \\
b^{4}-3\end{array}$ & $\mid \begin{array}{l}a^{2} b e+8 \\
a^{2} c d-12 \\
a b^{2} d-20 \\
a b c^{2}+36 \\
b^{3} c-12\end{array}$ & $\begin{array}{l}a^{2} c e+12 \\
a^{2} d^{2}-12 \\
a b^{2} e-8 \\
a b c d+12 \\
b^{3} d+4\end{array}$ & $\begin{array}{l}a^{2} c e-6 \\
a b^{2} e+30 \\
a b c d-48 \\
a c^{3}+54 \\
b^{3} d-48 \\
b^{2} c^{2}+18\end{array}$ & $\begin{array}{l}a b c e+60 \\
a b d^{2}-72 \\
a c^{2} d+36 \\
b^{3} e-36 \\
b^{2} c d+12\end{array}$ & $\begin{array}{l}a^{2} d e-4 \\
a b c e-12 \\
a b d^{2}+16 \\
a c^{2} d+36 \\
b^{3} e+48 \\
b^{2} c d-192 \\
b c^{3}+108\end{array}$ & $\begin{array}{l}a^{2} e^{2}+2 \\
a b d e-16 \\
a c^{2} e+36 \\
a c d^{2}-18 \\
b^{2} c e-18 \\
b^{2} d^{2}+14\end{array}$ & $\begin{array}{l}a^{2} e^{2}-4 \\
a b d e+20 \\
a c^{2} e+36 \\
b^{2} d^{2}-160 \\
b c^{2} d+108\end{array}$ \\
\hline
\end{tabular}

\begin{tabular}{c|c|c|c|c|c|c|}
\hline \multicolumn{1}{c|}{$T_{1}{ }^{4}$} & $T_{0} T_{1} T_{2}{ }^{2}$ & $T_{1}{ }^{3} T_{2}$ & $T_{0} T_{2}{ }^{3}$ & $T_{1}{ }^{2} T_{2}{ }^{2}$ & $T_{1} T_{2}{ }^{3}$ & $T_{2}{ }^{4}$ \\
\cline { 1 - 5 }$a^{2} e^{2}+1$ & $a c d e+60$ & $a b e^{2}-4$ & $a c e^{2}+12$ & $a c e^{2}-6$ & $a d e^{2}+8$ & $a e^{3}+1$ \\
$a b d e-16$ & $a d^{3}-36$ & $a c d e-12$ & $a d^{2} e-8$ & $a d^{2} e+30$ & $b c e^{2}-12$ & $b d e^{2}-4$ \\
$a c^{2} e-18$ & $b^{2} d e-72$ & $a d^{3}+48$ & $b^{2} e^{2}-12$ & $b c d e-48$ & $b d^{2} e-20$ & $c d^{2} e+6$ \\
$a c d^{2}+48$ & $b c^{2} e+36$ & $b^{2} d e+16$ & $b c d e+12$ & $b d^{3}-48$ & $c^{2} d e+36$ & $d^{4}-3$ \\
$b^{2} c e+48$ & $b c d^{2}+12$ & $b c^{2} e+36$ & $b d^{3}-4$ & $c^{3} e+54$ & $c d^{3}-12$ & \\
$b c^{2} d-144$ & & $b c d^{2}-192$ & & $c^{2} d^{2}+18$ & & \\
$c^{4}+81$ & & $c^{3} d+108$ & & & & \\
\hline
\end{tabular}

J'écris

$$
\begin{aligned}
& U^{\prime}=a T_{0}^{2}+4 b T_{0} T_{1}+c\left(2 T_{0} T_{2}+4 T_{1}^{2}\right)+4 d T_{1} T_{2}+e T_{2}^{2} \\
& \begin{aligned}
\dot{H}^{\prime}=\left(a c-b^{2}\right) T_{0}^{2}+2(a d-b c) T_{0} T_{1}+(a e-2 b d & \left.+c^{2}\right) T_{0} T_{2}+4\left(b d-c^{2}\right) T_{1}^{2} \\
& +2(b e-c d) T_{1} T_{2}+\left(c e-d^{2}\right) T_{2}^{2},
\end{aligned}
\end{aligned}
$$


et je représente par $4 \Phi^{\prime}$ la valeur qui vient d'être trouvée pour $\mathfrak{D}$. Ces expressions $U^{\prime}, H^{\prime}, \Phi^{\prime}$ sont des invariants des deux formes $(a, b, c, d, e \gamma \xi, \eta)^{4},\left(T_{0}, T_{1}, T_{2} \gamma \eta,-\xi\right)^{2}$, on a de plus les invariants

$$
a e-4 b d+3 c^{2}, \quad a c e-a d^{2}-b^{2} e+2 b c d-c^{3},
$$

que je représente comme à l'ordinaire par $I$, $J$, et l'invariant $T_{0} T_{2}-T_{1}{ }^{2}$ que je représente par $\Theta^{\prime}$. Cela posé on a

$$
\begin{aligned}
& \mathfrak{\complement}=6 H^{\prime}-2 I \Theta^{\prime}, \\
& \mathfrak{D}=4 \Phi^{\prime}, \\
& \left(\check{E}=I U^{\prime 2}-3 H^{\prime 2}+I^{2} \Theta^{\prime 2}+12 J \Theta^{\prime} U^{\prime}+2 I \Theta^{\prime} H^{\prime} .\right.
\end{aligned}
$$

La dernière de ces équations peut être vérifiée aisément, pour cela on a seulement besoin de remarquer qu'en posant $a=e=1, b=d=0, c=\theta$, elle devient

$$
\begin{aligned}
& \left(1+3 \theta^{2}\right)\left(T_{0}^{2}+\theta\left(2 T_{0} T_{2}+4 T_{1}^{2}\right)+T_{2}^{2}\right)^{2} \\
& -3\left(\theta T_{0}^{2}+\left(1+\theta^{2}\right) T_{0} T_{2}-4 \theta^{2} T_{1}^{2}+\theta T_{2}^{2}\right)^{2} \\
& +\left(1+3 \theta^{2}\right)^{2}\left(T_{0} T_{2}-T_{1}^{2}\right)^{2} \\
& +12\left(\theta-\theta^{3}\right)\left(T_{0} T_{2}-T_{1}^{2}\right)\left(T_{0}^{2}+\theta\left(2 T_{0} T_{2}+4 T_{1}^{2}\right)+T_{2}^{2}\right) \\
& +2\left(1+3 \theta^{2}\right)\left(T_{0} T_{2}-T_{1}^{2}\right)\left(\theta T_{0}^{2}+\left(1+\theta^{2}\right) T_{0} T_{2}-4 \theta^{2} T_{1}^{2}+\theta T_{2}^{2}\right) \\
& =T_{0}^{4} \\
& +T_{0}^{3} T_{2}(12 \theta) \\
& +T_{0}{ }^{2} T_{1}^{2}\left(-6 \theta+54 \theta^{3}\right) \\
& +T_{0}^{2} T_{2}^{2}\left(2+36 \theta^{2}\right) \\
& +T_{0} T_{1}^{2} T_{2}\left(-4+36 \theta^{2}\right) \\
& +T_{1}^{4}\left(1-18 \theta^{2}+81 \theta^{4}\right) \\
& +T_{1}^{2} T_{2}^{2}\left(-6 \theta+54 \theta^{3}\right) \\
& +T_{0} T_{2}^{3}(12 \theta) \\
& +T_{2}^{4}
\end{aligned}
$$

équation qui est identique. L'expression de l'invariant $I$ (quadrinvariant) de la fonction

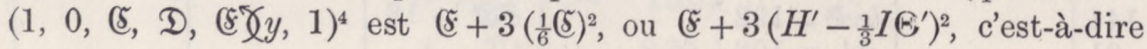

$$
\begin{array}{rr}
I U^{\prime 2}-3 H^{\prime 2}+I^{2} \Theta^{\prime 2}+12 J \Theta^{\prime} U^{\prime}+2 I \Theta^{\prime} H^{\prime} \\
+3 H^{\prime 2}+\frac{1}{3} I^{2} \Theta^{\prime 2} & -2 I \Theta^{\prime} H^{\prime},
\end{array}
$$

ou enfin

$$
I U^{\prime 2}+\frac{4}{3} I^{2} \Theta^{\prime 2}+12 J \Theta^{\prime} U^{\prime}
$$


ce qui est égal à

$$
\frac{1}{I}\left[\left(I U^{\prime}+6 J \Theta^{\prime}\right)^{2}+\frac{4}{3}\left(I^{3}-27 J^{2}\right) \Theta^{\prime 2}\right]
$$

La condition à remplir pour que cet invariant se réduise à zéro peut donc être présentée sous la forme

$$
I U^{\prime}+\left[6 J \pm 2 \sqrt{-\frac{1}{3}\left(I^{3}-27 J^{2}\right)}\right] \Theta^{\prime}=0,
$$

ce qui s'accorde avec un résultat trouvé par M. Hermite.

Il doit y avoir, ce me semble, une équation identique de la forme

$$
J U^{\prime 2}-I U^{\prime 2} H^{\prime}+4 H^{\prime 3}+M \Theta^{\prime}=-\Phi^{\prime 2}
$$

qui servirait à exprimer le carré de $\Phi^{\prime}$ au moyen des autres invariants $U^{\prime}, H^{\prime}, \Theta^{\prime}, I, J$, mais en supposant que cette équation existe, la forme du facteur $M$, que je n'ai pas encore cherchée, reste à déterminer; l'invariant $J$ (cubinvariant) de la forme

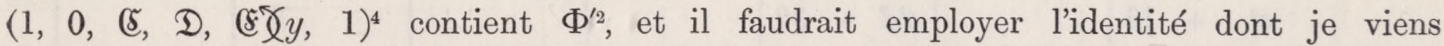
de parler pour réduire à sa forme la plus simple cet invariant; dans l'état actuel de la question je ne m'occupe donc pas de l'expression du cubinvariant de $(1,0, \mathfrak{E}, \mathfrak{D}, \mathfrak{E})(y, 1)^{4}$.

Pour passer au cas d'une équation du cinquième ordre, on devra faire usage de la formule qui se rapporte à la forme $\left(a, b, c, d, e^{\gamma} x, 1\right)^{4}$. En faisant la substitution nécessaire on arrive à ce résultat que pour l'équation

$$
\left(a, b, c, d, e^{\gamma}(x, 1)^{4}=0\right.
$$

la transformée en

$$
y=\left(a x+\frac{1}{4} b\right) T_{0}+\left(a x^{2}+b x+\frac{1}{2} c\right) T_{1}+\left(a x^{3}+b x^{2}+c x+\frac{3}{4} d\right) T_{2}
$$

\begin{tabular}{|c|c|c|c|c|c|c|c|c|c|}
\hline$T_{0}^{3}$ & $T_{0}^{2} T_{1}$ & $T_{0}^{2} T_{2}^{\prime}$ & $T_{0} T_{1}^{2}$ & $T_{0} T_{1} T_{2}$ & $T_{1}^{3}$ & $T_{0} T_{2}^{2}$ & $T_{1}^{2} T_{2}$ & $T_{1} T_{2}^{2}$ & $T_{2}^{3}$ \\
\hline$=\frac{1}{8} \quad \begin{array}{l}a^{2} d+8 \\
a b c-4 \\
b^{3}+1\end{array}$ & $\begin{array}{l}a^{2} e+32 \\
a b d+4 \\
a c^{2}-8 \\
b^{2} c+2\end{array}$ & $\begin{array}{l}a b e+8 \\
a c d-4 \\
b^{2} d+1\end{array}$ & $\begin{array}{l}a b e+32 \\
a c d-16 \\
b^{2} d+4\end{array}$ & $\begin{array}{l}a d^{2}-12 \\
b^{2} e+12\end{array}$ & $\begin{array}{l}a d^{2}-8 \\
b^{2} e+8\end{array}$ & $\begin{array}{l}a d e-8 \\
b c e+4 \\
b d^{2}-1\end{array}$ & $\begin{array}{l}a d e-32 \\
b c e+16 \\
b d^{2}-4\end{array}$ & $\begin{array}{l}a e^{2}-32 \\
b d e-4 \\
c^{2} e+8 \\
c d^{2}-2\end{array}$ & $\begin{array}{l}b e^{2}-8 \\
c d e+4 \\
d^{3}-1\end{array}$ \\
\hline
\end{tabular}

est la suivante

$$
\left(1,0,\left(5, D,(E)(y, 1)^{4}=0,\right.\right.
$$

où

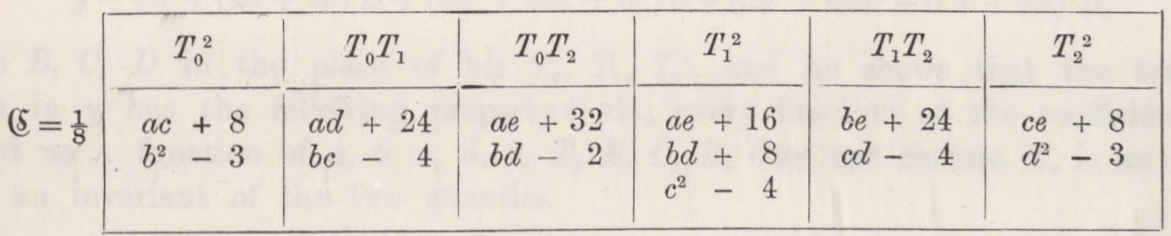




\begin{tabular}{|c|c|c|c|c|c|c|c|c|}
\hline & $T_{0}^{4}$ & $T_{0}^{3} T_{1}$ & $T_{0}^{3} T_{2}$ & $T_{0}^{2} T_{1}^{2}$ & $T_{0}^{2} T_{1} T_{2}$ & $T_{0} T_{1}^{3}$ & $T_{0}^{2} T_{2}^{2}$ & $T_{0} T_{1}^{2} T_{2}$ \\
\hline$=\frac{1}{256}$ & $\begin{array}{l}a^{3} e+256 \\
a^{2} b d-64 \\
a b^{2} c+16 \\
b^{4}-3\end{array}$ & $\begin{array}{l}a^{2} b e+512 \\
a^{2} c d-128 \\
a b^{2} d-80 \\
a b c^{2}+64 \\
b^{3} c-8\end{array}$ & $\begin{array}{l}a^{2} c e+512 \\
a^{2} d^{2}-192 \\
a b^{2} e-128 \\
a b c d+32 \\
b^{3} d-4\end{array}$ & $\begin{array}{l}a^{2} c e-256 \\
a b^{2} e+480 \\
a b c d-128 \\
a c^{3}+64 \\
b^{3} d-48 \\
b^{2} c^{2}+8\end{array}$ & $\begin{array}{l}a b c e+640 \\
a b d^{2}-288 \\
a c^{2} d+64 \\
b^{3} e-144 \\
b^{2} c d+8\end{array}$ & $\begin{array}{l}a^{2} d e-256 \\
a b c e-128 \\
a b d^{2}+64 \\
a c^{2} d+64 \\
b^{3} e+192 \\
b^{2} c d-128 \\
b c^{3}+32\end{array}$ & $\begin{array}{l}a^{2} e^{2}+512 \\
a b d e-256 \\
a c^{2} e+256 \\
a c d^{2}-48 \\
b^{2} c e-48 \\
b^{2} d^{2}+14\end{array}$ & $\mid \begin{array}{l}a^{2} e^{2}-1024 \\
a b d e+320 \\
a c^{2} e+256 \\
b^{2} d^{2}-160 \\
b c^{2} d+48\end{array}$ \\
\hline
\end{tabular}

\begin{tabular}{|c|c|c|c|c|c|c|}
\hline$T_{1}^{4}$ & $T_{0} T_{1} T_{2}^{2}$ & $T_{1}^{3} T_{2}$ & $T_{0} T_{2}^{3}$ & $T_{1}^{2} T_{2}^{2}$ & $T_{1} T_{2}^{3}$ & $T_{2}^{4}$ \\
\hline $\begin{array}{l}a^{2} e^{2}+256 \\
a b d e-256 \\
a c^{2} e-128 \\
a c d^{2}+128 \\
b^{2} c e+128 \\
b c^{2} d-64 \\
c^{4}+16\end{array}$ & $\begin{array}{l}a c d e+640 \\
a d^{3}-144 \\
b^{2} d e-288 \\
b c^{2} e+64 \\
b c d^{2}+8\end{array}$ & $\begin{array}{l}a b e^{2}-256 \\
a c d e-128 \\
a d^{3}+192 \\
b^{2} d e+64 \\
b c^{2} e+64 \\
b c d^{2}-128 \\
c^{3} d+32\end{array}$ & $\begin{array}{l}a c e^{2}+512 \\
a d^{2} e-128 \\
b^{2} e^{2}-192 \\
b c d e+32 \\
b d^{3}-4\end{array}$ & $\begin{array}{l}a c e^{2}-256 \\
a d^{2} e+480 \\
b c d e-128 \\
b d^{3}-48 \\
c^{3} e+64 \\
c^{2} d^{2}+8\end{array}$ & $\begin{array}{l}a d e^{2}+512 \\
b c e^{2}-128 \\
b d^{2} e-80 \\
c^{2} d e+64 \\
c d^{3}-8\end{array}$ & $\begin{array}{lr}a e^{3}+256 \\
b d e^{2}-64 \\
c d^{2} e+16 \\
d^{4}-3\end{array}$ \\
\hline
\end{tabular}

En m'appuyant sur ce résultat j'espère être à même de trouver la formule pour l'équation du cinquième ordre.

Londres, 11 Mai 1860. 